\title{
Correspondence
}

\section{Step up funding for flu prevention}

Experts on pandemic influenza need to convince governments and the public that, although the 2009-10 'swine flu' pandemic proved to be relatively mild, the threat posed by future flu pandemics is severe and warrants more investment in public education and research (Nature 482, 131; 2012).

Without effective education, there is a danger that the low mortality in the 2009-10 pandemic will exacerbate public scepticism over the lethality of flu. This could undermine compliance with basic preventative measures, such as conscientious personal hygiene and social isolation.

Research funding for pandemic flu prevention and treatment must be stepped up. The US government spent US\$26.2 billion on HIV/AIDS activities in 2010, a year in which 1.8 million people died from AIDS worldwide. Tuberculosis killed a comparable number in 2009 and received $\$ 224$ million for research from the US National Institutes of Health alone in 2010. A pandemic strain of flu could, in theory, cause up to 60 million deaths worldwide in just two years. The $\$ 627$ million that the US government spent on flu research in 2008-10 (see go.nature.com/c2wwke) would then seem decidedly insufficient. Simon N. Williams

Northwestern University, Chicago, Illinois, USA.

simonwilliams@northwestern.edu

\section{Keep jellyfish numbers in check}

It may be unclear whether jellyfish numbers are rising globally (Nature 482, 20-21; 2012), but this should not distract us from taking urgent action to control populations in those degraded ecosystems where particular species have undeniably increased.
You mention the "paradigm" that jellyfish are increasing globally, but this consensus view is not held by the scientific community because jellyfish time series are scarce. Even with new database syntheses of scientific data, supplemented by anecdotal information from fishermen, news reports, historical research cruises and modelled reconstructions of historic ecosystems, we are unlikely to reach a consensus in the near future.

There is compelling evidence that some jellyfish species pose a risk in particular marine systems, so we believe that precautionary action should be taken now. Efforts could focus on increasing surveillance (currently scanty for jellyfish) and minimizing habitat eutrophication, overfishing and species translocation, all thought to cause jellyfish outbreaks.

Anthony J. Richardson CSIRO Marine and Atmospheric Research, Dutton Park, Queensland; and University of Queensland, St Lucia, Australia. anthony.richardson@csiro.au Daniel Pauly University of British Columbia, Vancouver, Canada.

Mark J. Gibbons University of the Western Cape, Bellville, South Africa.

\section{Clinical standards not practical in the lab}

Gholson Lyon calls for more rigorous standards in genetics research so that results can be disclosed to subjects and their families as valid clinical diagnostic information (Nature 482, 300-301; 2012). This well-intentioned proposal is impractical because it would add huge costs for only occasional benefit.

Lyon is essentially recommending that the standards of Good Clinical Practice (see go.nature.com/1pado7) should apply to laboratory research as well. The cost and complexity of carrying out all research to comply with these regulations would be crippling. Compliance would demand validated environments for sampletracking (Lyon's focus) and for all analytical work. In whole-genome sequencing, validation for error-free determination of those 3 billion or so data points in the human genome would be almost impossible.

A more pragmatic alternative would be to incorporate specific provisions in the informed consent for people wanting to access their data. This would carry the disclaimer that results are not formally validated for clinical use, which would require independent replication to meet the standards of Good Clinical Practice. This re-testing could be made available at no cost to the patient should the clinical need arise.

The cost of responding to such an occasional requirement would be insignificant in comparison with processing every subject's specimens under regulatorycompliant conditions.

Klaus Lindpaintner SDIX, Newark, Delaware, USA; and Bio-Banks Consulting, Basel, Switzerland.

klindpaintner@sdix.com

\section{Sugar: an excess of anything can harm}

As director-general of the World Sugar Research Organisation, I wish to point out some shortcomings in the latest discussion of sugar's impact on health (Nature 482, 27-29; 2012).

Robert Lustig and colleagues incorrectly say that sugar consumption has tripled worldwide since the 1960 s. The global population has more than doubled in that time, so the increase in sugar supply per head is more like $60 \%$. In fact, the United States, Canada and the United Kingdom all show only marginal changes over the past few decades in average sugar consumption as

a proportion of food-energy intake.

The authors argue that sugar can kill because of its supposed influence on metabolic syndrome (itself a controversial concept), indirectly implicating a WHO Technical Report that draws no such conclusion. There is little consistent effect on the symptoms of this syndrome in people who eat up to three times more sugar than the average Western intake (A. S. Truswell Am. J. Clin. Nutr. 59, 710S-718S; 1994). Neither have any deaths been attributed to dietary sugars in an exhaustive analysis of US mortality figures (G. Danaei et al. PLoS Medicine 6, e100058; 2009).

The Food and Agriculture Organization of the United Nations, the US Food and Nutrition Board, and the European Food Standards Authority have all considered the issues now revisited by Lustig et al. and find no reliable evidence that typical sugar consumption contributes to any disease apart from dental caries. Without evidence that reducing sugar consumption would improve public health, Lustig and colleagues' policy proposals are irrelevant.

Scientific controversies should be settled by consideration of all the available evidence, not of a seemingly biased selection. Overconsumption of anything is harmful, including of water and air.

Richard C. Cottrell World Sugar Research Organisation, London, UK.rcottrell@wsro.org Competing financial interests declared (see go.nature.com/ ek29gr).

CONTRIBUTIONS

Correspondence may be sent to correspondence@ nature.com after consulting the guidelines at http:// go.nature.com/cmchno. Alternatively, readers may comment online: www.nature.com/nature. 